\title{
How the Observation in a New Custom Basis Based on Orbital Angular Momentum Space Could Improve Our Dose Effective Reconstruction of the Protein Structure.
}

Filippo Troiani ${ }^{1}$, Enzo Rotunno ${ }^{2}$, Stefano Frabboni ${ }^{3}$, Raimond Ravelli ${ }^{4}$, Peter Peters ${ }^{4}$, Ebrahim Karimi $^{5}$ and Vincenzo Grillo ${ }^{1}$

${ }^{1}$ CNR-Istituto di Nanoscienze, Modena, Emilia-Romagna, Italy, ${ }^{2}$ CNR-NANO, Modena, EmiliaRomagna, Italy, ${ }^{3}$ Università di Modena e Reggio Emilia, Modena, Emilia-Romagna, Italy, ${ }^{4}$ Maastricht University, Maastricht, Limburg, Netherlands, ${ }^{5}$ University of Ottawa, Ottawa, Ontario, Canada

One of the most difficult challenge in microscopy is to observe protein due to dose restriction imposed by the damage of the sample. Normally characterizing the single protein can be very complicated so that many images of different proteins must be collected.

In the imaging of a single protein it is therefore essential to restrict the amount of information that must be extracted. In particular we reduce the problem to discriminate macromolecular structures in an electron microscope, through a specific beam shaping technique [1]. Our approach is based on maximizing the which-molecule information extracted from the state of each electron. To this aim, the optimal observables can be derived within the framework of" quantum state discrimination".

In this framework we can obtain 1) the maximum discrimination probability based on the scalar product of the electron wave, of the beam passing through the proteins 0,1 [2].

2) that the use of an Orbital Angular Momentum (OAM) sorter [3] permits to remove the loss of information due to the ignorance of the protein in plane orientation. 3) That a projective measurement of the radial degree of freedom on an optimal state given by a combination of the two proteins permits to reach the theoretical maximum discrimination with the minimum number of electrons.

This idea provides a new experimental framework for cryomicroscopy of single proteins. The new instrument should be an extension of an OAM sorter comprising a third additional phase element where a programmable phase plate can be chosen for the projective measurement of the radial degree of freedom. The scheme of the optics is shown in fig 1 . An example of discrimination is visible in fig 2 . Thanks to the versatility of the programmable phase plate we can also imagine the integration with deep learning algorithm elaborating in real time the best guess for the optimal phase element.

This work is supported by Q-SORT, a project funded by the European Union's Horizon 2020 Research and Innovation Program under grant agreement No. 766970. 


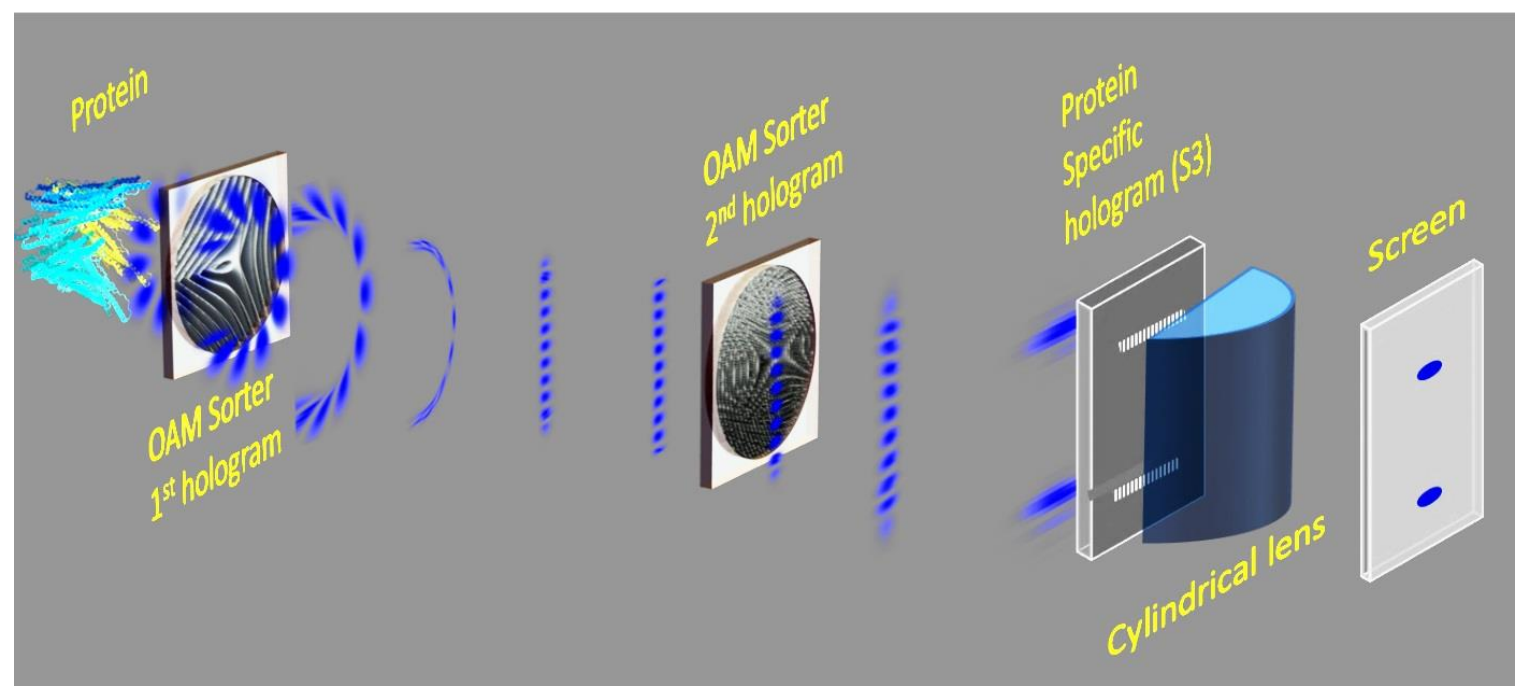

Figure 1. Schematic view of the generalized OAM sorter. With respect to the standard OAM sorter, a third phase element is added. The structure of such third element is adapted to the radial structure of the proteins to be recognized.
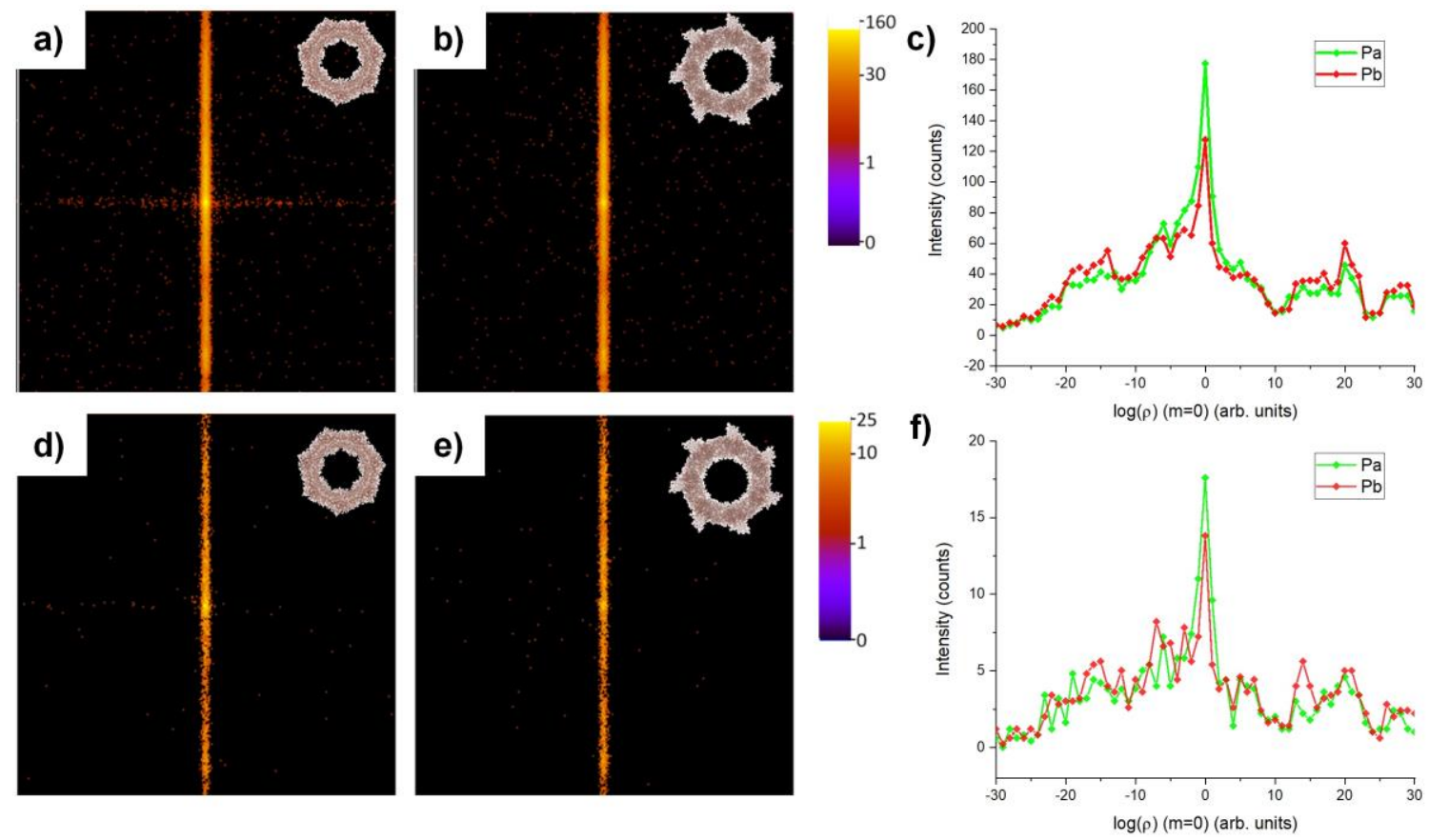

Figure 2. Example of discrimination between 2 proteins. The upper and the lower panels correspond respectively to doses of $2 \mathrm{e}^{-} / \AA^{2}(\mathrm{a}, \mathrm{b})$ and $0.2 \mathrm{e}^{-} / \AA^{2}(\mathrm{~d}, \mathrm{e})$. The proteins $1(\mathrm{a}, \mathrm{d})$ and $2(\mathrm{~b}, \mathrm{e})$ give rise to different distributions of the detected electrons in the correlated angular-radial basis. (c,f) Statistics of the radial observable corresponding to the $m=0$ subspace.

\section{References}

[1] F Troiani, E Rotunno et al. arXiv:2001.08918

[2] C. W. Helstrom, Quantum Detection and Estimation Theory (Academic Press, New York, 1976).

[3] V. Grillo, A. H. Tavabi et al. Nat. Comm. 8, 15536 (2017). 